\title{
NOTE ON A RADICAL OF DIVINSKY
}

\author{
by A. D. SANDS \\ (Received 20th March 1987)
}

In his book [1] Divinsky refers to eight radicals as classical. In [6] radicals were considered such that the radical of each one-sided ideal of a ring may be expressed as the intersection of a left ideal and a right ideal of the ring. From results obtained there it was deduced that seven of these eight radicals have this property. The purpose of this note is to give a proof that this property also holds for the remaining one of these classical radicals.

Divinsky [1] defines this radical to be the lower radical generated by the nil radicals of rings with descending chain condition. Thus it is the smallest radical to coincide with the classical radical on rings with d.c.c. By using results of Fuchs [2], Gardner [3] has shown that a ring $R$ belongs to this radical $\mathscr{D}$ of Divinsky if and only if $R$ belongs to the lower Baer radical $\beta$ and also $R / T$ is divisible, as an additive abelian group, where $T$ is the maximal torsion subgroup of $R$. Thus if $\delta$ denotes the divisible radical, $\tau$ the torsion radical and $\alpha$ is the lower radical generated by $\delta \cup \tau$ then $\mathscr{D}=\beta \cap \alpha$. Since $\delta$ and $\tau$ are $A$-radicals it follows that $\alpha$ is an $A$-radical [4]. Hence $\mathscr{D}$ is a normal radical and so is left and right strong and principally left and right hereditary [5].

It is shown in [6, Theorem 8] that, for any $A$-radical $\alpha$, the radical $\beta \cap \alpha$ has the desired property if and only if $R \in \alpha$ implies $\beta(R) \in \alpha$. We shall prove that this property holds for the above radical $\alpha$.

Let $R \in \alpha$ and let $S=R / \alpha(\beta(R))$. Then $S \in \alpha$ and $\beta(S)=\beta(R) / \alpha(\beta(R))$ belongs to $\mathscr{S}_{\alpha}$, where $\mathscr{S}_{\alpha}$ denotes the semisimple class of the radical $\alpha$. Thus to show that $R \in \alpha$ implies $\beta(R) \in \alpha$ it suffices to show that $R \in \alpha, \beta(R) \in \mathscr{S}_{\alpha}$ implies $\beta(R)=0$.

So now let $R$ be a ring with $R \in \alpha$ and $\beta(R) \in \mathscr{S}_{\alpha}$. Let $\tau(R)=T$ be the maximal torsion subgroup of $R$. Then $\beta(T) \in \alpha$ and $\beta(R) \in \mathscr{S}_{\alpha}$ implies $\beta(T)=0$. For each prime $p$ let $T_{p}$ denote the $p$-component of $T$ and $T[p]=\{x \in T \mid p x=0\}$. Then, as $T[p] p R=0$, $T[p] \cap p R$ is a nilpotent ideal of $T . \beta(T)=0$ then implies $T[p] \cap p R=0$. So $p^{2} x=0$ implies $p x \in T[p] \cap p R=0$. It follows that $T_{p}=T[p]$. Since $R / T$ is divisible we have $T_{p}+p R=R$. Now $T=\sum T_{q}$, where the summation is taken over all primes $q$. For $q \neq p$ we have $T_{q}=p T_{q} \subseteq p R$. It follows that $T[p]+p R=R$. $T[p] \cap p R=0$ then implies $\beta(R)=\beta(T[p])+\beta(p R)=\beta(p R)$. Also $p R=p T[p]+p^{2} R=p^{2} R$. Hence, by Lemma 16 of [4], applied to $p R$ we have $\beta(p R)=p \beta(p R)$. This gives $\beta(R)=p \beta(R)$ and so $\beta(R)$ is divisible. Thus $\beta(R) \in \alpha$. However $\beta(R) \in \mathscr{S}_{\alpha}$ and so $\beta(R)=0$, as required. 


\section{A. D. SANDS}

\section{REFERENCES}

1. N. J. Divinsky, Rings and Radicals (Toronto University Press, Toronto, 1965).

2. L. Fuchs, Ringe und ihre additive Gruppe, Publ. Math. Debrecen 4 (1956), 488-508.

3. B. J. Gardner, Some remarks on radicals of rings with chain conditions, Acta Math. Acad. Sci. Hungar. 25 (1974), 263-268.

4. M. JAEgermann and A. D. SAnds, On normal radicals, $N$-radicals and $A$-radicals, $J$. Algebra 50 (1978), 337-349.

5. A. D. SANDs, On normal radicals, J. London Math. Soc. 11 (1975), 361-365.

6. A. D. Sands, Radicals and one-sided ideals, Proc. Royal Soc. Edinburgh 103 (1986), 241-251.

Department of Mathematics and Computer Science

UNIVERSITY OF DUNDEE

DUNDEE, DDI 4HN

SCOTLAND 